\title{
Editorial
}

\section{Risk of Infections in Patients with Chronic Diseases}

\author{
Anil Mor ${ }^{\S}$ and Reimar W. Thomsen ${ }^{*}, \S$
}

Department of Clinical Epidemiology, Institute of Clinical Medicine, Aarhus University Hospital, Aarhus, Denmark

Keywords: Chronic disease, epidemiological transition, epidemiology, infection, risk.

The industrialized world is facing what some have called the third epidemiological transition [1]: related to long life expectancy and unhealthy lifestyle factors, an increasing prevalence of chronic diseases is accompanied by revival of many familiar infections [2,3] caused by increasingly resistant microorganisms. Chronic non-communicable diseases (NCDs) [4] such as diabetes, cardiovascular disease, chronic respiratory disease, and cancer currently sweep the entire globe [5,6]. Diet and lifestyle related diseases are no longer "diseases of the rich" but emerge in developing countries at a fast rate [7], before the battle against poverty and under-nutrition has been won [8]. The risk of many infections may be increased in people with chronic diseases, and developing countries' health care systems are put under stress with a double burden of communicable and noncommunicable diseases [9-11]. Clinical epidemiological studies play a key role in clarifying the association of chronic conditions with susceptibility to and outcome of infectious diseases. Such research studies form the basis for our understanding of disease mechanisms and clinical course, and thus for primary, secondary and tertiary prevention of infections in people with chronic disease around the globe. The objective of this special issue of The Open Infectious Disease Journal is to review the existing knowledge on the risk of infections in patients with a number of important chronic diseases.

Diabetes now affects approximately 346 million people worldwide and it was liable for $6.8 \%$ of global deaths in 2010 [12]. Thomsen \& Mor review recent epidemiological evidence that community-acquired infections are a major clinical and public health problem in people with diabetes. For example, the risk of a major killer - pneumonia appears to be $30 \%$ to $50 \%$ increased with diabetes, whereas the risk of tuberculosis may be 1.5 to 3 times increased in settings with poor glycemic control and in low-income countries. Corroborating clinical experience, the relative risk from diabetes is particularly high for urinary tract infections and for severe staphylococcal and streptococcal infections. Important risk factors include impaired neutrophil and

*Address correspondence to this author at the Department of Clinical Epidemiology, Institute of Clinical Medicine, Aarhus University Hospital, Olof Palmes Alle 43-45, DK-8200 Aarhus N, Denmark;

Tel: +45 8716 8204; Fax: +45 8716 7215; E-mail: rwt@dce.au.dk

${ }^{\S}$ Guest Editors macrophage function, obesity, late diabetes complications, and metabolic effects of hyperglycemia. Good glycemic control, lifestyle interventions, improved patient and caregiver education, and relevant vaccinations are recommended to reduce infection risk.

Every year, approximately 15 million people suffer an episode of severe stroke worldwide, out of them 6 million die and 5 million suffer permanent disability [13]. Infections are a leading cause of death in the acute hospital admission phase of patients with stroke. Johnsen and colleagues discuss the risk of post-stroke pneumonia and urinary tract infections and their consequences in adult stroke patients. They discuss the underlying mechanisms including immobility, aspiration, invasive procedures and brain-induced immunosuppression. The authors argue that preventive use of antibiotics, reduced use of permanent catheters, and better organized in-patient care can significantly reduce the risk of infections in such patients.

Patients with hematological malignancies constitute only a fraction of cancer patients but may be those at the highest risk of infection [14]. Both the immunosuppression caused by the malignancy itself and the neutropenia induced by intensive chemotherapeutic treatment contribute to this risk. Nørgaard concisely reviews current data on a number of important infections in adults with hematological malignancies. She describes the challenges in identifying infection in these patients due to compromised inflammatory symptoms, and discusses the important role of blood culture reports, central venous catheters, changes in the spectrum of microorganisms, and emergence of antimicrobial resistance. Nørgaard advocates a strict antimicrobial strategy for infection prophylaxis to reduce the prevalence of resistant microorganisms.

Acute exacerbations of chronic obstructive pulmonary disease (COPD) caused by infection play an important role in making COPD the $5^{\text {th }}$ leading cause of death worldwide [15]. Wackerhausen \& Hansen discuss the various inflammatory mechanisms and microorganisms that may be involved in COPD progression and exacerbation; mechanisms that are still incompletely understood. The authors go on reviewing the risk of respiratory infections in patients with COPD and also include remarks on nonrespiratory infections, while discussing the important role of COPD severity and use of corticosteroids. There seems to be good evidence that antibiotics improve outcomes following 
COPD exacerbation, whereas the role of antibiotics for prevention of exacerbations is promising but still controversial. Further good data are needed.

The skin is our major physiological barrier to microorganisms. In patients with chronic skin diseases the epidermal barrier function is disrupted, and skin diseases may indeed be the most common of all chronic conditions discussed in this special issue. Olesen reviews the biological mechanisms that lead to infections in patients with chronic skin disease. She then discusses evidence for the risk of different bacterial, viral and fungal infections, including staphylococcal infections, molluscum contagiosum, eczema herpeticum, dermatophytes and candida infection. Importantly, patients with chronic skin disease may also be prone to systemic infections; for example, children with atopic dermatitis may have increased risk of respiratory tract infections, and psoriasis patients seem to have an increased mortality due to infections.

The five reviews in this special issue indicate that patients with chronic diseases are at generally increased risk of infections, yet much still needs to be learned about the underlying biological mechanisms. Doctors and policymakers need understandings of the exact mechanisms to effectively intervene against risk factors [16], and thereby to improve prevention and therapy of infections in people with chronic diseases. We hope this special issue of The Open Infectious Diseases Journal will act as a catalyst for ongoing studies and will be a motivation for future studies, the results of which will hopefully contribute to decrease risk and improve prognosis of infections in patients with chronic diseases.

\section{REFERENCES}

[1] Harper K, Armelagos G. The changing disease-scape in the third epidemiological transition. Int J Environ Res Public Health 2010; 7(2): 675-97.

[2] Søgaard M, Nørgaard M, Dethlefsen C, Schonheyder HC. Temporal changes in the incidence and 30-day mortality associated with bacteremia in hospitalized patients from 1992 through 2006: a population-based cohort study. Clin Infect Dis 2011; 52(1): 61-9.

[3] Thomsen RW, Riis A, Norgaard M, et al. Rising incidence and persistently high mortality of hospitalized pneumonia: a 10-year population-based study in Denmark. J Intern Med 2006; 259(4): 410-7.

[4] The NCD Alliance. NCD Alliance Report 2009-2011. Available at: http: //ncdalliance.org/Report2011 [Cited 2012 July 12].

[5] Haines A, Alleyne G, Kickbusch I, Dora C. From the Earth Summit to Rio+20: integration of health and sustainable development. Lancet 2012; 379(9832): 2189-97.

[6] Beaglehole R, Yach D. Globalisation and the prevention and control of non-communicable disease: the neglected chronic diseases of adults. Lancet 2003; 362(9387): 903-8.

[7] Hall V, Thomsen RW, Henriksen O, Lohse N. Diabetes in Sub Saharan Africa 1999-2011: epidemiology and public health implications. A systematic review. BMC Public Health 2011; 11: 564.

[8] Vorster HH, Kruger A, Margetts BM. The nutrition transition in Africa: can it be steered into a more positive direction? Nutrients 2011; 3(4): 429-41.

[9] Boutayeb A. The double burden of communicable and noncommunicable diseases in developing countries. Trans R Soc Trop Med Hyg 2006; 100(3): 191-9.

[10] Meyrowitsch DW, Bygbjerg IC. Global burden of disease--a race against time. Dan Med J 2007; 54(1): 32-4.

[11] Dooley KE, Chaisson RE. Tuberculosis and diabetes mellitus: convergence of two epidemics. Lancet Infect Dis 2009; 9(12): 737 46.

[12] Roglic G, Unwin N. Mortality attributable to diabetes: estimates for the year 2010. Diabetes Res Clin Pract 2010 ; 87(1): 15-9.

[13] World Health Organization. Global Burden of Stroke. Available at: http: //www.who.int/cardiovascular_diseases/resources/atlas/en/. [Cited 2012 July 12]

[14] Norgaard M, Larsson H, Pedersen G, Schonheyder HC, Sorensen HT. Risk of bacteraemia and mortality in patients with haematological malignancies. Clin Microbiol Infect 2006; 12(3): 217-23.

[15] World Health Organization. Burden of COPD. Available at: http: //www.who.int/respiratory/copd/burden/en/index.html [Cited 2012 July 12].

[16] Jones DS, Podolsky SH, Greene JA. The burden of disease and the changing task of medicine. N Engl J Med 2012; 366(25): 2333-8.

Mor and Thomsen; Licensee Bentham Open.

This is an open access article licensed under the terms of the Creative Commons Attribution Non-Commercial License (http: //creativecommons.org/licenses/by$\mathrm{nc} / 3.0 /$ ) which permits unrestricted, non-commercial use, distribution and reproduction in any medium, provided the work is properly cited. 